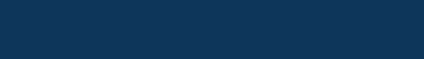

\title{
Excess mortality in Finnish diabetic subjects due to alcohol, accidents and suicide: a nationwide study
}

\author{
Leo Niskanen1, Timo Partonen², Anssi Auvinen³ and Jari Haukka ${ }^{4}$ \\ ${ }^{1}$ Endocrinology and Diabetology, Abdominal Center, Helsinki University Hospital and the Universities of Helsinki and \\ Eastern Finland, Helsinki, Finland, ${ }^{2}$ Department of Public Health Solutions, National Institute for Health and Welfare \\ (THL), Helsinki, Finland, ${ }^{3}$ Epidemiology/Health Sciences, Faculty of Social Sciences, University of Tampere, Tampere, \\ Finland, and ${ }^{4}$ Department of Public Health, University of Helsinki, Helsinki, Finland
}

Correspondence

should be addressed

to L Niskanen

Email

leo.niskanen@hus.fi

\begin{abstract}
Aims: To characterize the burden of external causes of death attributable to alcohol-related causes, accidents and suicide among diabetic patients in a large national cohort.

Methods: The population included diabetic individuals who had purchased and received reimbursement for at least one insulin prescription and/or one oral antidiabetic drug prescription between January 1997 and December 2010, and a non-diabetic reference population matched by sex, age and area. All new insulin users in this period were included, as well as $50 \%$ of new oral drug (OAD) users as a random sample. The data were collected by means of linkage from national registries. We analyzed the cohort data using Poisson regression models separately for each end-point and by gender (mortality rate ratio (MRR)). We subjected the case-cohort data to conditional logistic regression analysis based on exposure information within 1 year of the end-point event. The follow-up started on the date of the first diabetes medication prescription and ended on 31 December 2012 or on the date of death.

Results: The study population comprised 434629 individuals (226 372 men; diabetes population: 208148 subjects, of whom $76 \%$ were treated only with OAD). The mean follow-up time was 7.1 years, during which there were 2832 deaths attributable to alcohol, 3187 to accidents and 853 to suicide. The diabetic subjects had higher mortality at almost all end-points, especially those treated with insulin: the adjusted MRRs for alcohol-related deaths were 1.71 for diabetic men treated with OAD and 6.92 for those on insulin; the respective MRRs for diabetic women were 2.10 and 10.60. There were more accident-related deaths among those treated with insulin (MRRs: 2.06 and 1.53 for men and women, respectively), and more suicides (MRR: 2.10 for men treated with insulin and 1.62 among women treated only with $O A D)$. The results from the cohort study and the case-cohort study were rather similar. A time-dependent effect of diabetes was observed in alcohol-related mortality among men.

Conclusions: The findings from this large nationwide cohort indicate higher mortality attributable to suicide, alcoholrelated causes and accidents among diabetic patients than among the non-diabetic population. The results emphasize the importance of effective psychosocial interventions among high-risk diabetic patients.
\end{abstract}

European Journal of

Endocrinology

(2018) 179, 299-306

\section{Introduction}

Patients with diabetes mellitus are known to have markedly higher mortality attributable to cardiovascular diseases, cancer and renal causes, whereas the mental (c) 2018 European Society of Endocrinology Printed in Great Britain strain imposed by chronic diseases and substance abuse as well as accidental causes of excess mortality have attracted relatively little attention. 
In particular, diabetic patients treated with daily injections of insulin have a heavy, unremitting burden: how they eat, move and sleep affect plasma glucose levels. Miscalculation or omission in terms of self-care exposes the patient to hypoglycemia, and consequent neuroglycopenic symptoms up to the loss of consciousness may increase the risk of accidental death. Mental symptoms and diseases such as depression and suicidal ideation are common among diabetic patients $(1,2,3)$. However, in cases of type 2 diabetes, the relationship with depression may be bidirectional: depression increases the risk of diabetes (4) and the concomitant depression hampers its treatment via multiple mechanisms (2). It was found in a recent meta-analysis (5) and a national cohort study from Sweden (6) that diabetic subjects showed a significantly increased risk of suicide, especially those with insulin-deficient (type 1) diabetes.

Excess alcohol consumption is a major contributor to the burden of diseases and to mortality, and it is associated with an increased risk of accidental and suicidal causes of death (7). Among diabetic patients, it is also associated with an increased risk of severe hypoglycemia (8). Suicide, alcohol-related and accidental causes of death are often interwoven and therefore their simultaneous assessment gives a broader view on the fatality burden associated with diabetes.

For this report, we analyzed mortality attributable to alcohol-related causes, accidents and suicide in a large national cohort including all insulin-treated diabetic patients and a 50\% random sample of those treated with $\mathrm{OAD}$, as well as a matched control group from the general population.

\section{Subjects and methods}

\section{Study population}

The choice of subjects was based on the CARING Project $(9,10)$ being conducted in Finland. The study population consisted of two groups. Group 1, comprising diabetes mellitus (DM) patients, included individuals who had purchased and received reimbursement for at least one insulin prescription (ATC code A10A) (11) and/or one oral antidiabetic drug (OAD) prescription (ATC A10B) between January 1, 1997 and December 31, 2010. All individuals in the diabetes treatment group were new cases, because we required 1 year without any diabetes drug before the start of the follow-up. The year 1996 was the initial wash-in period, meaning that it was guaranteed that no individual in the study population had been given a prescription within 1 year before the start of the follow-up. Group 2, the reference (Ref) group, was individually matched to the DM group by sex, age (same birth year) and region (hospital district). All insulin users in Finland during this period were included, as well as a 50\% random sample of OAD users. The population comprised 434629 individuals (Table 1). There were 13199 individuals in the reference cohort who started diabetes medication during the follow-up. After that, they were treated as new cases: a new matched control was assigned to each and a matched non-diabetic subject was added to the reference group. Unfortunately, some irregularities were detected in the matching of data obtained from the Social Insurance Institution (SII); hence, the study population was constructed by the authors using original raw data on prescriptions, age, sex and location. Thus, the original matching pairs were not used in the analyses hence the slightly different population sizes of the DM and reference groups. However, balance in terms of sex, age and location distributions between groups was maintained.

We obtained the prescription data from SII (permission Kela 16/522/2012). Cancer data were obtained from the Finnish Cancer Registry (12), and included diagnosis (ICD-O-3 code) (13) and the date of diagnosis (THL/264/5.05.00/2012). This is a register-based study with anonymous data and no patient contact, thus no consents from anonymized patients were required according to Finnish law.

The Ethical Committee of the Faculty of Medicine, University of Helsinki, Finland (02/2012) reviewed the protocol. Data permits were received from the Social Insurance Institute (SII) (16/522/2012), the National Institute for Health and Welfare (THL/264/5.05.00/2012) and Statistics Finland (TK-53-214-12). SII anonymized the data.

\section{Follow-up}

The follow-up started on the date of the first diabetes medication prescription and ended on December 31, 2012 or on the date of death, whichever occurred first. If an individual in the reference group received a diabetes prescription during the follow-up, a new reference individual was assigned, and the exposure status was updated and changed from the reference to the diabetes group.

We obtained the information on mortality (the date and cause of death) from Statistics Finland (permission TK-53-214-12) (14). 
Table 1 Basic characteristics of study population in start of follow-up, number of subjects and percentage in parentheses.

\begin{tabular}{|c|c|}
\hline \multirow[t]{2}{*}{ Sex } & Male \\
\hline & Female \\
\hline \multirow[t]{9}{*}{ Age } & {$[0,10]$} \\
\hline & $(10,20]$ \\
\hline & $(20,30]$ \\
\hline & $(30,40]$ \\
\hline & $(40,50]$ \\
\hline & $(50,60]$ \\
\hline & $(60,70]$ \\
\hline & $(70,80]$ \\
\hline & $(80,100]$ \\
\hline \multirow[t]{14}{*}{ Start of follow-up } & $(1997,1998]$ \\
\hline & $(1998,1999]$ \\
\hline & $(1999,2000]$ \\
\hline & $(2000,2001]$ \\
\hline & $(2001,2002]$ \\
\hline & $(2002,2003]$ \\
\hline & $(2003,2004]$ \\
\hline & $(2004,2005]$ \\
\hline & $(2005,2006]$ \\
\hline & $(2006,2007]$ \\
\hline & $(2007,2008]$ \\
\hline & $(2008,2009]$ \\
\hline & $(2009,2010]$ \\
\hline & $(2010,2011]$ \\
\hline \multirow[t]{2}{*}{ Cancer } & No \\
\hline & Yes \\
\hline \multirow[t]{2}{*}{ Statin } & No \\
\hline & Yes \\
\hline \multirow[t]{2}{*}{ HRT (females) } & No \\
\hline & Yes \\
\hline
\end{tabular}

$\begin{array}{r}\hline \text { No } \\ \hline 117527(51.9 \%) \\ 108954(48.1 \%) \\ 4546(2.0 \%) \\ 4491(2.0 \%) \\ 5624(2.5 \%) \\ 11138(4.9 \%) \\ 22062(9.7 \%) \\ 51077(22.6 \%) \\ 56745(25.1 \%) \\ 46996(20.8 \%) \\ 23802(10.5 \%) \\ 12014(5.3 \%) \\ 11005(4.9 \%) \\ 10964(4.8 \%) \\ 11897(5.3 \%) \\ 12195(5.4 \%) \\ 12899(5.7 \%) \\ 13596(6.0 \%) \\ 15728(6.9 \%) \\ 15791(7.0 \%) \\ 16015(7.1 \%) \\ 22016(9.7 \%) \\ 25745(11.4 \%) \\ 23657(10.4 \%) \\ 22959(10.1 \%) \\ 219530(96.9 \%) \\ 6951(3.1 \%) \\ 193448(85.4 \%) \\ 33033(14.6 \%) \\ 86393(79.3 \%) \\ 22561(20.7 \%) \\ \hline\end{array}$

Note: '(' is an open interval, and '[' is a closed interval. $O A D$, oral antidiabetic drugs.

\section{End-points and other variables}

We used death with an underlying cause from suicide and sequelae of intentional self-harm (ICD-10 X60-X84, Y870), accident (V01-X44, X46-X59, Y85-Y86, Y10-Y15) and alcohol-related diseases and accidental poisoning by alcohol (F10, G312, G4051, G621, G721, I426, K292, K70, K860, K852, 0354, P043, Q860, X45) as the end-point events.

We had information on the following prescriptions (ATC, date, amount of purchased medications in DDD) (11): oral antidiabetic drugs (OAD) (A10), HMG CoA reductase inhibitors (C10AA) (statins), estrogens (G03C) and progesterone and estrogens in combination (G03F). We categorized subjects into four groups by the use of diabetes medication at the start of the follow-up: no DM, only OAD, OAD and insulin and only insulin. Statin and hormone replacement therapy (HRT) (no, yes) at baseline were defined using prescriptions issued within one year before the start of the follow-up. A cancer diagnosis before

the baseline (no/yes) was determined using data from the Finnish Cancer Registry.

To enable us to study risk factors within 1 year before the end-point event we constructed case-cohort data separately for each three end-points, and with three controls for each case from the whole study population in the follow-up (15). In these data, the cases and the controls were matched by sex, age, calendar year of the start of follow-up and hospital district. We analyzed the case-cohort design using variables based on the data within 1 year before the end-point event.

\section{Statistical methods}

We analyzed the cohort data using Poisson regression models separately for each end-point, and for men and women. The following variables were used in the models: diabetes group, age-group (as 10-year categories), calendar year at the start of the follow-up, usage of statins at 
Table 2 Mortality in diabetic population compared to reference population by gender.

\begin{tabular}{|c|c|c|c|c|}
\hline & & $\begin{array}{c}\text { P-years } \\
(1000)\end{array}$ & Deaths & $\begin{array}{c}\text { Mortality (per } 1000 \\
P \text {-years) }\end{array}$ \\
\hline \multicolumn{5}{|c|}{ Alcohol related } \\
\hline \multirow[t]{4}{*}{ Males } & No & 777 & 714 & $0.92(0.85,0.99)$ \\
\hline & OAD only & 680 & 1052 & $1.55(1.45,1.64)$ \\
\hline & OAD + Insulin & 23 & 66 & $2.90(2.24,3.69)$ \\
\hline & Insulin only & 120 & 480 & $4.00(3.65,4.37)$ \\
\hline \multirow[t]{4}{*}{ Females } & No & 742 & 137 & $0.18(0.15,0.22)$ \\
\hline & OAD only & 641 & 254 & $0.40(0.35,0.45)$ \\
\hline & OAD + Insulin & 12 & 12 & $1.01(0.52,1.77)$ \\
\hline & Insulin only & 107 & 117 & $1.10(0.91,1.32)$ \\
\hline \multicolumn{5}{|l|}{ Accidents } \\
\hline \multirow[t]{4}{*}{ Males } & No & 777 & 938 & $1.21(1.13,1.29)$ \\
\hline & OAD only & 680 & 877 & $1.29(1.21,1.38)$ \\
\hline & OAD + Insulin & 23 & 35 & $1.54(1.07,2.14)$ \\
\hline & Insulin only & 120 & 160 & $1.33(1.13,1.56)$ \\
\hline \multirow[t]{4}{*}{ Females } & No & 742 & 542 & $0.73(0.67,0.79)$ \\
\hline & OAD only & 641 & 568 & $0.89(0.81,0.96)$ \\
\hline & OAD + Insulin & 12 & 24 & $2.03(1.30,3.02)$ \\
\hline & Insulin only & 107 & 43 & $0.40(0.29,0.54)$ \\
\hline \multicolumn{5}{|l|}{ Suicides } \\
\hline \multirow[t]{4}{*}{ Males } & No & 777 & 273 & $0.35(0.31,0.40)$ \\
\hline & OAD only & 680 & 272 & $0.40(0.35,0.45)$ \\
\hline & OAD + Insulin & 23 & 15 & $0.66(0.37,1.09)$ \\
\hline & Insulin only & 120 & 77 & $0.64(0.51,0.80)$ \\
\hline \multirow[t]{4}{*}{ Females } & No & 742 & 81 & $0.11(0.09,0.14)$ \\
\hline & OAD only & 641 & 108 & $0.17(0.14,0.20)$ \\
\hline & OAD + Insulin & 12 & 6 & $0.51(0.19,1.10)$ \\
\hline & Insulin only & 107 & 21 & $0.20(0.12,0.30)$ \\
\hline
\end{tabular}

\begin{tabular}{ccc}
\hline \multicolumn{1}{c}{ Unadjusted } & & Adjust \\
\cline { 1 - 1 } MRR & MRR \\
(Reference) & & (Reference) \\
$1.68(1.53,1.85)$ & & $1.71(1.56,1.89)$ \\
$3.15(2.45,4.06)$ & & $2.86(2.22,3.68)$ \\
$4.35(3.88,4.88)$ & & $6.92(6.14,7.80)$ \\
(Reference) & & (Reference) \\
$2.15(1.74,2.64)$ & & $2.10(1.70,2.58)$ \\
$5.49(3.05,9.91)$ & & $4.79(2.65,8.65)$ \\
$5.95(4.65,7.61)$ & & $10.60(8.14,13.79)$
\end{tabular}

(Reference)

$1.07(0.97,1.17)$

$1.27(0.91,1.78)$

$1.10(0.93,1.31)$

(Reference)

$1.21(1.08,1.36)$

$2.78(1.85,4.18)$

$0.55(0.40,0.75)$

(Reference)

$1.14(0.96,1.35)$

$1.88(1.12,3.15)$

$1.83(1.42,2.35)$

(Reference)

$1.54(1.16,2.06)$

$4.65(2.03,10.65)$

$1.81(1.12,2.92)$
(Reference)

$1.04(0.94,1.14)$

$1.29(0.92,1.81)$

(Reference)

$1.12(0.99,1.26)$

$2.42(1.60,3.64)$

$1.53(1.11,2.11)$

(Reference)

$1.14(0.96,1.35)$

$1.68(1.00,2.82)$

$2.10(1.60,2.75)$

(Reference)

$1.62(1.21,2.17)$

$4.49(1.95,10.31)$

$1.49(0.89,2.50)$
$2.06(1.73,2.46)$
OR

(Reference)

$1.19(1.04,1.35)$

$2.44(2.03,2.92)$

$6.88(5.83,8.11)$

(Reference)

$1.73(1.29,2.32)$

$3.45(2.26,5.25)$

$14.97(9.81,22.85)$

(Reference)

$0.98(0.87,1.11)$

$1.48(1.23,1.79)$

$2.09(1.71,2.54)$

(Reference)

$1.09(0.93,1.28)$

$1.82(1.39,2.38)$

$1.51(1.10,2.07)$

(Reference)

$1.03(0.82,1.31)$

$1.54(1.11,2.14)$

$2.78(2.01,3.84)$

(Reference)

$1.96(1.33,2.88)$

$1.57(0.84,2.94)$

$1.76(0.92,3.37)$

Mortality rates with $95 \%$ confidence intervals, unadjusted mortality rate ratios (MRR) and adjusted MRR from cohort study, and odds ratios (OR) from case-cohort study for end-points with $95 \%$ confidence intervals. Adjusted MRR estimated by Poisson regression and adjusted for age, calendar year in start of follow-up, usage of statins, usage of HRT, time since start of follow-up, cancer before start of follow-up and hospital district. OR estimated by conditional logistic regression and adjusted for statin and HRT use and cancer.

$O A D$, oral antidiabetic drugs.

baseline, usage of HRT at baseline, time since entry, cancer before baseline and hospital district (23 districts). The case-cohort data were analyzed by means of conditional logistic regression stratified by the matching factors.

The results from the Poisson regression models are reported as mortality rate ratios (MRR), and those for the case-cohort study as odds ratios (OR). We used Aalen's linear regression model to analyze changes of risk in the treatment groups during the follow-up (16). All the results are reported with $95 \%$ confidence intervals. The analyses were based on the R data-analysis language (17) and the Epi package (18).

\section{Results}

The study population consisted of 434629 individuals: 226372 men and 208257 women (Table 1). The diabetes population comprised 208148 subjects, of whom 76\% were treated only with OAD. The mean follow-up time was 7.1 years. We observed 2832 deaths attributable to alcohol-related causes (1981 in the diabetic subjects), 3187 from accidents (1707 in the diabetic subjects) and 853 from suicide (499 in the diabetic subjects) (Table 2). The most frequent alcohol-related cause of death was alcoholic cirrhosis of the liver (ICD $10 \mathrm{~K} 70.3$; 45\% of all deaths). There were no substantial differences between the diabetes treatment groups in this regard (Supplementary Table 1, see section on supplementary data given at the end of this article). The most frequent accidental cause of death (36\%) was a fall on some level from slipping, tripping or stumbling (W01; Supplementary Table 2). The most frequent cause of death by suicide was intentional self-harm by hanging, strangulation or suffocation (X70), accounting for 30\% of all deaths (Supplementary Table 3 ). The second most frequent suicide method (17\%) was intentional self-poisoning by antiepileptic, sedativehypnotic, antiparkinsonism and psychotropic drugs, not elsewhere classified (X61). Diabetic individuals were more likely to use this suicide method. 


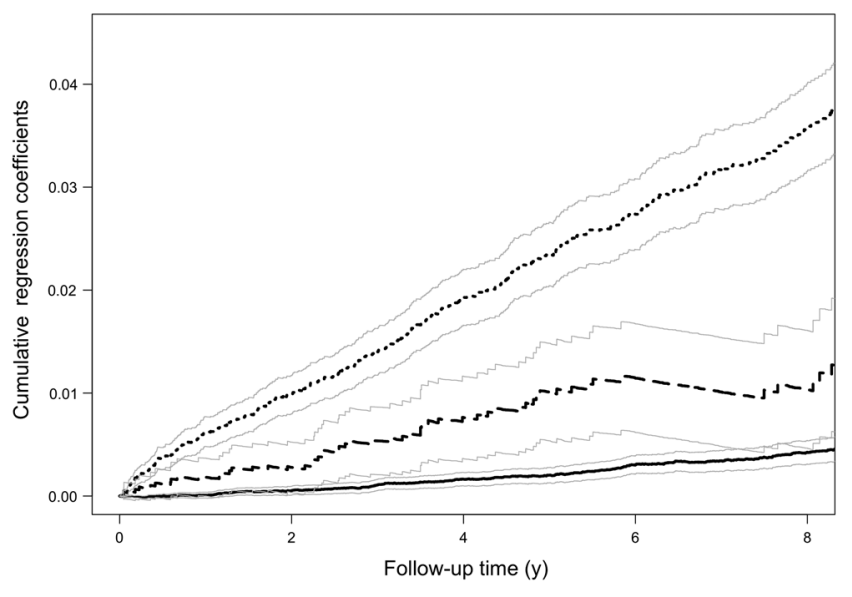

Figure 1

Alcohol-related mortality among men; cumulative regression coefficients with $95 \%$ confidence intervals based on Aalen's model: only OAD (continuous line), OAD and insulin (dashes), only insulin (dots) compared to the reference group. Adjusted for age, calendar period, cancer and statin usage.

The unadjusted and adjusted analyses revealed consistently higher alcohol-related mortality among diabetic patients in all treatment groups and in both genders (Table 2). Although the mortality rates in this category were lower among the women than among the men, the unadjusted and adjusted MRRs were higher among the women (from 2.10 to 10.60 vs from 1.68 to 6.92 among the men). With regard to accidents, the adjusted MRRs were higher among men and women in the insulin-treated group (2.06 for men and 1.53 for women treated with insulin only; 2.42 for women treated with OAD+insulin). Suicide mortality was substantially lower among women than among men (MRR: 0.35, 95\% CI: 0.30-0.41). Men with diabetes treated with OAD faced a similar suicide risk as men without diabetes, whereas the risk was somewhat higher among men taking OAD+insulin (adjusted MRR: 1.68; 1.00, 2.82 of borderline significance), and markedly higher in the insulin-only treatment group (MRR: 2.10; 1.60, 2.75). There was a higher suicide risk among diabetic women (MRR: $1.62 ; 1.21,2.17$ ) in the OAD-only group and among those taking OAD+insulin $(4.49 ; 1.95,10.31)$. However, the increased risk in the insulin-only group attenuated to a non-significant level after adjustment (1.49; 0.89, 2.50), as well as in the case-cohort setting.

In general, the results from the cohort study and the case-cohort study were similar (Table 2). The largest difference was between the MRRs and the ORs among the insulin-only group in the case of alcohol-related deaths.
The odds ratio in the case-control study was about double that in the cohort study. The probable interpretation is that a recent (during the previous year) diabetes status is clearly a better predictor of mortality than diabetes at baseline (further back in the patient's medical history).

We evaluated changes in mortality across the follow-up time using Aalen's regression model. The only clear time-dependent effect of diabetes was in alcoholrelated mortality among men (Fig. 1): mortality increased more quickly in the insulin-only group than in the other treatment groups. Aalen's regression reveals any differences in incidence rates in relation to the follow-up time.

\section{Discussion}

The risk of suicide, and alcohol-related and accidental death was elevated in this nationwide cohort among diabetic subjects compared with their matched controls. In particular, male diabetic subjects and those treated with insulin were at the highest risk, but in the case of alcohol-related deaths, female subjects with diabetes showed excess mortality regardless of treatment modality.

Suicide is not an uncommon cause of death globally, affecting about one million people annually. Chronic major diseases such as diabetes predispose to mentalhealth problems, but psychiatric illness as clinical comorbidity has received relatively little attention in guidelines on diabetes treatment (19). Studies assessing the risk of suicide among diabetic individuals have yielded controversial findings. It was found in a recent comprehensive systematic review and meta-analysis that the overall risk of suicide was markedly increased among diabetic individuals (RR: 16; 95\% CI: 1.29-1.89) and was especially pronounced in patients with type 1 diabetes (RR: 2.25; 95\% CI: 1.50-3.38) (5). The risk among those with type 2 diabetes appeared to be increased (RR: 1.65; 95\% CI: 0.95-2.85), but this estimate was based on quite a small number of subjects, and a further problem was the apparent misclassification of intoxication and drug poisoning. Thus, suicide risk among diabetes patients is probably underestimated, and more large-scale population-based studies are needed.

Intentional overdoses of insulin may underlie acute admissions for severe hypoglycemia of 'unknown' etiology, and this may also lead to the underestimation of suicide as a cause of death among patients with diabetes (20). However, the majority of suicides among the diabetic subjects in this study were attributable to psychotrophic drugs, as in the Swedish study (6) in which 
the use of insulin or hypoglycemic agents was identified as contributing to suicide in nine per cent of the cases. Nevertheless, according to the above-mentioned metaanalysis (5), the incidence ratio of suicide among diabetics was 2.85 per 10000 person-years, on the basis of which it is estimated that globally there are about 94000 suicides each year among diabetic individuals. In our study, female patients treated with OAD emerged as a risk group in terms of suicide. Although this finding is somewhat difficult to explain, diabetes-related distress is particularly pronounced among females with type 2 diabetes, and the relationship between depression and type 2 diabetes is bidirectional (3).

A high proportion of alcohol- and drug-related deaths was found in a nationwide Finnish study comprising a large cohort of patients with type 1 diabetes (diagnosed before the age of 30 years) (21). One in four deaths was associated with alcohol use or drugs in an early-onset cohort (diagnosis at the age of $0-14$ years). In a late-onset cohort (diagnosis at the age of 15-29 years); however, there was a conspicuous increase in such deaths among those diagnosed after 1985. The standardized mortality ratio for alcohol-related deaths was 1.5 , and alcohol use or drugs accounted for $39 \%$ of the deaths (21). Furthermore, the standardized mortality ratio for ischemic heart disease was up to 17.4 (12.6-22.1) in the early-onset cohort, which is attributable to the rare occurrence of death from ischemic heart disease among young people in the general population (21).

Heavy drinking is associated with clinical depression (22). About $25 \%$ of patients with type 1 diabetes investigated in a large Finnish survey were moderate or heavy consumers of alcohol, and they also showed a higher frequency of microvascular diabetic complications (23). Not only does excessive alcohol consumption negatively affect diabetes self-care adherence, it also affects the course of the illness and increases the risk of severe hypoglycemia via multiple mechanisms. Alcohol consumption among patients with diabetes is frequently inadequately assessed in the first place, and neglected thereafter in everyday medical care (8).Validated brief interventions to reduce risky drinking are available and offer the potential to improve adherence to diabetes treatment, and thereby probably the outcome as well (24). Accidental causes of death were likewise higher among the diabetic patients in our study. One obvious explanation is treatmentrelated hypoglycemia, and hypoglycemia is known to increase the risk of accidents. Other causes may have included microvascular or macrovascular complications, which predispose to accidents via multiple mechanisms, including impaired vision, gait unsteadiness, tiredness and cardiac problems. However, we could not assess the role of complications in this study. As discussed, some accidental deaths may also be suicides.

The strengths of this study include the large nationwide sample and the assessment of three groups of causes of death, which are usually overshadowed by an emphasis on cardiovascular and diabetic complications. Studies on mortality among diabetic individuals should be population-based. In many countries, however, the information on death certificates does not allow the identification of patients with diabetes, especially with type 2 diabetes (25).

On the other hand, among the weaknesses of our study is the lack of access to information on the use of antidepressant and other psychoactive drugs among the participants. It has been shown previously that the use of antidepressants is associated with a lowered (26) risk of death from suicide in the general population. It was found among a large cohort of patients with type 1 diabetes in which purchases of antidepressants were used as a marker of depression (27) that about eight per cent of the patients had bought them. Additional cases were observed during the follow-up increasing the percentage to 18 , and in the adjusted Cox's regression models, the purchase of antidepressants at baseline was associated with mortality among women, but not among men. Among those purchasing antidepressants, chronic diabetic complications were the most frequent underlying cause of death and only a small minority was suicides. The men in our study had a higher risk for every accidental cause of death, and it is conceivable that those seeking professional help for depression or other mental disorder are more problematic. We adjusted for the use of statins and HRT because these medications are suspected risk factors for cancer (9) (the original end-point of the CARING study), and the information was obtainable from the SII database. In addition, their use is indicative of health-service patronage, and probably of health awareness in general, related in part to socioeconomic status.

Another weakness of our study is that we could only assess the type of diabetes in accordance with the type of therapy. However, those treated only with insulin comprise type 1 diabetics and some long-term type 2 patients whose disease could be classified as insulinrequiring, and the demarcation between the types of diabetes in individual cases may also be rather arbitrary in clinical practice. A third problem is that we were not able to assess socioeconomic status or the presence of diabetic complications, or their contributions to the increased mortality. Finally, the follow-up was relatively short. 
To conclude, we found in this large nationwide cohort that patients with diabetes have higher mortality attributable to suicide, alcohol-related causes and accidents than the rest of the population and that this premature mortality is associated with insulin use, which reflects the severity of the illness. This effect was most consistent among diabetic men, but in cases of alcoholrelated death female diabetic patients showed excess mortality regardless of treatment modality. These findings underscore the importance of effective psychosocial support and interventions in diabetes, especially among high-risk individuals.

\section{Supplementary data}

This is linked to the online version of the paper at https://doi.org/10.1530/ EJE-18-0351.

\section{Declaration of interest}

The authors declare that there is no conflict of interest that could be perceived as prejudicing the impartiality of this study.

\section{Author contribution statement}

All the authors have made substantial contributions: conception and design (all), data acquisition and analysis $(\mathrm{J} \mathrm{H}, \mathrm{A} \mathrm{A})$, data interpretation (all). They all participated in drafting the article or revising it critically for important intellectual content, and all have approved the final version.

\section{Acknowledgements}

The authors would like to thank the CARING study (EU grant agreement number 282526) group for their co-operation during the project (28) that made these data available to the authors ( $\mathrm{A} A$ and $\mathrm{J} \mathrm{H}$ are members of the CARING group).

\section{References}

1 Peyrot M, Rubin RR, Lauritzen T, Snoek FJ, Matthews DR \& Skovlund SE. Psychosocial problems and barriers to improved diabetes management: results of the Cross-National Diabetes Attitudes, Wishes and Needs (DAWN) Study. Diabetic Medicine 2005 22 1379-1385. (https://doi.org/10.1111/j.1464-5491.2005.01644.x)

2 Petrak F, Baumeister H, Skinner TC, Brown A \& Holt RIG. Depression and diabetes: treatment and health-care delivery. Lancet Diabetes and Endocrinology 20153 472-485. (https://doi.org/10.1016/S22138587(15)00045-5)

3 Snoek FJ, Bremmer MA \& Hermanns N. Constructs of depression and distress in diabetes: time for an appraisal. Lancet Diabetes and Endocrinology 20153 450-460. (https://doi.org/10.1016/S22138587(15)00135-7)

4 Moulton CD, Pickup JC \& Ismail K. The link between depression and diabetes: the search for shared mechanisms. Lancet Diabetes and Endocrinology 20153 461-471. (https://doi.org/10.1016/S22138587(15)00134-5)

5 Wang B, An X, Shi X \& Zhang J. Management of endocrine disease: suicide risk in patients with diabetes: a systematic review and metaanalysis. European Journal of Endocrinology 2017177 R169-R181. (https://doi.org/10.1530/EJE-16-0952)
6 Webb RT, Lichtenstein P, Dahlin M, Kapur N, Ludvigsson JF \& Runeson B. Unnatural deaths in a national cohort of people diagnosed with diabetes. Diabetes Care 201437 2276-2283. (https:// doi.org/10.2337/dc14-0005)

7 Rehm J, Gmel GE, Gmel G, Hasan OSM, Imtiaz S, Popova S, Probst C, Roerecke M, Room R, Samokhvalov AV et al. The relationship between different dimensions of alcohol use and the burden of disease - an update. Addiction 2017112 968-1001. (https://doi. org/10.1111/add.13757)

8 Engler PA, Ramsey SE \& Smith RJ. Alcohol use of diabetes patients: the need for assessment and intervention. Acta Diabetologica 201350 93-99. (https://doi.org/10.1007/s00592-010-0200-x)

9 CAncer Risk and INsulin analoGues Common Study Protocol. (available at: http://www.encepp.eu/encepp/viewResource. htm?id=9633).

10 But A, Bruin MLD, Bazelier MT, Hjellvik V, Andersen M, Auvinen A, Starup-Linde J, Schmidt MK, Furu K, de Vries F et al. Cancer risk among insulin users: comparing analogues with human insulin in the CARING five-country cohort study. Diabetologia 201760 1691-1703. (https://doi.org/10.1007/s00125-017-4312-5)

11 Anonym. ATC/DDD Index 2006. 2006. (available at: http://www. whocc.no/atc_ddd_index/).

12. Leinonen MK, Miettinen J, Heikkinen S, Pitkäniemi J \& Malila N. Quality measures of the population-based Finnish Cancer Registry indicate sound data quality for solid malignant tumours. European Journal of Cancer 201777 31-39. (https://doi.org/10.1016/j. ejca.2017.02.017)

13 WHO. International Classification of Diseases for Oncology, 3rd ed. (ICD-O-3). (available at: http://www.who.int/classifications/icd/ adaptations/oncology/en/).

14 Lahti R. \& Penttilä A. The validity of death certificates: routine validation of death certification and its effects on mortality statistics. Forensic Science International 2001115 15-32. (https://doi. org/10.1016/S0379-0738(00)00300-5)

15 Clayton D \& Hills M. Statistical Models in Epidemiology. Oxford, UK: Oxford Univ. Press, 1993.

16 Aalen OO. A linear regression model for the analysis of life times. Statistics in Medicine 19898 907-925.

17 R Core Team. R: A Language and Environment for Statistical Computing. Vienna, Austria: R Foundation for Statistical Computing, 2016.

18 Carstensen B, Plummer M, Laara E \& Hills M et al. Epi: A Package for Statistical Analysis in Epidemiology. 2008. https://www.pubhealth. $\mathrm{ku} . \mathrm{dk} / \mathrm{bxc} / \mathrm{Epi} /$

19 The Lancet Diabetes \& Endocrinology. Poor mental health in diabetes: still a neglected comorbidity. Lancet Diabetes and Endocrinology 20153 393. (https://doi.org/10.1016/S22138587(15)00144-8)

20 Russell KS, Stevens JR \& Stern TA. Insulin overdose among patients with diabetes: a readily available means of suicide. Primary Care Companion to the Journal of Clinical Psychiatry 200911 258-262. (https://doi.org/10.4088/PCC.09r00802)

21 Harjutsalo V, Forsblom C \& Groop PH. Time trends in mortality in patients with type 1 diabetes: nationwide population based cohort study. BMJ 2011343 d5364. (https://doi.org/10.1136/bmj.d5364)

22 Paljärvi T, Koskenvuo M, Poikolainen K, Kauhanen J, Sillanmäki L \& Mäkelä P. Binge drinking and depressive symptoms: a 5-year population-based cohort study. Addiction 2009104 1168-1178. (https://doi.org/10.1111/j.1360-0443.2009.02577.x)

23 Harjutsalo V, Feodoroff M, Forsblom C, Groop PH \& the FinnDiane Study Group. Patients with type 1 diabetes consuming alcoholic spirits have an increased risk of microvascular complications. Diabetic Medicine 201431 156-164. (https://doi.org/10.1111/ dme.12307)

24 Kaner EFS, Dickinson HO, Beyer F, Pienaar E, Schlesinger C, Campbell F, Saunders JB, Burnand B \& Heather N. The effectiveness of brief alcohol interventions in primary care settings: a systematic 
review. Drug and Alcohol Review 200928 301-323. (https://doi. org/10.1111/j.1465-3362.2009.00071.x)

25 Mühlhauser I, Sawicki P, Blank M, Overmann H, Richter B \& Berger M. Reliability of causes of death in persons with Type I diabetes. Diabetologia 200245 1490-1497. (https://doi.org/10.1007/ s00125-002-0957-8)

26 Haukka J, Arffman M, Partonen T, Sihvo S, Elovainio M, Tiihonen J, Lönnqvist J \& Keskimäki I. Antidepressant use and mortality in Finland: a register-linkage study from a nationwide cohort. European
Journal of Clinical Pharmacology 200965 715-720. (https://doi. org/10.1007/s00228-009-0616-9)

27 Ahola AJ, Harjutsalo V, Saraheimo M, Forsblom C \& Groop PH. Purchase of antidepressant agents by patients with type 1 diabetes is associated with increased mortality rates in women but not in men. Diabetologia 201255 73-79. (https://doi.org/10.1007/ s00125-011-2347-6)

28 General introduction. CARING. (available at: http://www.caringdiabetes.eu/). Accessed on 23 April 2015.

Received 23 April 2018

Revised version received 13 July 2018

Accepted 9 August 2018 